\title{
BMJ Open Protocol for the economic evaluation of the China Salt Substitute and Stroke Study (SSaSS)
}

Ka-Chun Li (D) , ${ }^{1}$ Maoyi Tian, ${ }^{1,2}$ Bruce Neal, ${ }^{1,3}$ Liping Huang, ${ }^{1}$ Jie Yu (D) , ${ }^{1,4}$ Yishu Liu, ${ }^{1}$ Xuejun Yin (D) , ${ }^{1}$ Xinyi Zhang, ${ }^{2}$ Yangfeng Wu, ${ }^{2,5}$ Nicole Li, ${ }^{6}$ Paul Elliott (D) , ${ }^{3}$ Lijing Yan, ${ }^{2,7}$ Darwin Labarthe, ${ }^{8}$ Zhixin Hao, ${ }^{2}$ Jing Xiangxian Feng, ${ }^{10}$ Jianxin Zhang, ${ }^{11}$ Yuhong Zhang, ${ }^{12}$ Ruijuan Zhang, ${ }^{13}$ Bo Zhou, ${ }^{9}$ Zhifang Li, ${ }^{10}$ Jixin Sun, ${ }^{11}$ Yi Zhao, ${ }^{12}$ Yan Yu, ${ }^{13}$ Lei Si (D) , ${ }^{1}$ Thomas Lung (D) ${ }^{1,14}$

\section{ABSTRACT}

Introduction Cardiovascular diseases (CVDs) are the leading causes of death and disability worldwide. Reducing dietary salt consumption is a potentially cost-effective way to reduce blood pressure and the burden of CVD. To date, economic evidence has focused on sodium reduction in food industry or processed food with blood pressure as the primary outcome. This study protocol describes the planned within-trial economic evaluation of a low-sodium salt substitute intervention designed to reduce the risk of stroke in China.

Methods and analyses The economic evaluation will be conducted alongside the Salt Substitute and Stroke Study: a 5-year large scale, cluster randomised controlled trial. The outcomes of interest are quality of life measured using the EuroQol-5-Dimensions and major adverse cardiovascular events. Costs will be estimated from a healthcare system perspective and will be sought from the routinely collected data available within the New Rural Cooperative Medical Scheme. Cost-effectiveness and cost-utility analyses will be conducted, resulting in the incremental cost-effectiveness ratio expressed as cost per cardiovascular event averted and cost per quality-adjusted life year gained, respectively.

Ethics and dissemination The trial received ethics approval from the University of Sydney Ethics Committee (2013/888) and Peking University Institutional Review Board (IRB00001052-13069). Informed consent was obtained from each study participant. Findings of the economic evaluation will be published in a peer-reviewed journal and presented at international conferences.

Trial registration number ClinicalTrials.gov Registry (NCT02092090).

\section{INTRODUCTION}

Cardiovascular diseases (CVDs) are the leading causes of death and disability worldwide, accounting for 17.8 million deaths and 35.7 million years lived with disability (YLDs) in 2017. ${ }^{12}$ Currently, ischaemic heart disease (IHD) and stroke are the top two causes of CVD-related health loss globally and this ranking is predicted to remain the same

\section{Strengths and limitations of this study}

- This study will provide policymakers with valuable economic evidence based on a large-scale, cluster randomised controlled trial.

- First economic evaluation assessing the effects of salt substitution on morbidity and mortality of cardiovascular disease rather than blood pressure.

- Routinely collected data from the New Rural Cooperative Medical Scheme will be used in estimation of the medical costs.

- A potential limitation is the adherence to salt substitute observed in the trial may not reflect the realworld situation.

by $2040 .{ }^{3}$ Specifically, it was estimated that IHD and all types of stroke caused 8.9 and 6.2 million deaths; 5.3 and 18.7 million YLDs in 2017, respectively. ${ }^{12}$ The global economic impact of CVD may be catastrophic in the absence of effective preventive interventions ${ }^{3}$ and finding cost-effective interventions, particularly in resource constrained lowincome and middle-income country settings, is imperative to reduce the burden of CVD.

High blood pressure is the leading risk factor for CVD-related morbidity and mortality and is one of the most important modifiable risk factors for CVD prevention. ${ }^{4}$ According to a recent systematic review and meta-analysis of large scale randomised controlled trials, ${ }^{5}$ every $10 \mathrm{~mm} \mathrm{Hg}$ reduction in systolic blood pressure can reduce $20 \%$ relative risk for all major cardiovascular events and reduce corresponding $17 \%$ for coronary heart disease, $27 \%$ for stroke and $13 \%$ for all-cause mortality.

It was estimated that high dietary intake of sodium caused 3 million deaths and 70 million disability-adjusted life years globally in $2017 .^{6}$ The positive effects of lowering 
dietary sodium intake on reduction of blood pressure are well documented. ${ }^{78}$ It is anticipated that lowering sodium could likely prevent CVD through reducing blood pressure, but there has been no high-quality randomised clinical trial examining the direct effects of sodium reduction on vascular events and the association between them continues to be debated. ${ }^{9}{ }^{10}$ On the other hand, increasing potassium intake also reduces blood pressure and prior studies in rural China have proven that using salt substitutes can lower blood pressure markedly. ${ }^{11} 12$ Therefore, the Salt Substitute and Stroke Study (SSaSS) is designed to investigate the joint effects of sodium reduction and potassium increase through a sodium-reduced, potassium-enriched salt substitute on the risk of stroke. To date, there are no economic evaluations that have been conducted alongside a trial that has been powered to detect changes in CVD events (fatal or non-fatal stroke, acute coronary syndrome) and CVD deaths. The economic evaluations that have so far been conducted have focused on sodium substitution in processed foods, ${ }^{13} 14$ and have looked at reductions in blood pressure primarily. ${ }^{1516}$ The health economic effects of salt substitution within daily home cooking on prevention of CVD events remain unclear. However, a recent modelling study suggested the blood pressure lowering effects of such a nationwide salt substitution intervention could prevent around 460000 CVD deaths each year in China, including 208000 due to stroke and 175000 due to heart disease. ${ }^{17}$ This paper presents the protocol for the economic evaluation of the SSaSS trial, which will address the question of whether using a sodium-reduced, potassium-enriched salt substitute is a cost-effective intervention for the prevention of fatal and non-fatal stroke, total major cardiovascular events and total mortality in a high-risk population, compared with the use of usual salt.

\section{METHOD}

\section{The SSaSS}

SSaSS is a 5-year large-scale cluster randomised trial, which involves 20996 individuals of 600 villages (five provinces) in rural China. Full details of the design and baseline characteristics of the trial have been published separately. ${ }^{18}$ The trial started in July 2014 and the end-oftrial follow-up was completed in August 2020.

\section{Study population}

Based on a high prevalence of hypertension and stroke and home cooking dietary pattern, a total of 600 villages in five provinces (Liaoning, Shanxi, Hebei, Ningxia and Shaanxi) in north China were selected for the trial. Two counties from each province were selected based on the prior collaboration and their willingness to participate and 60 villages in each of the 10 counties were chosen. In each village, about 35 individuals at high risk of stroke with either (1) a history of stroke; or (2) aged 60 years or older with uncontrolled high blood pressure; and (3) contactable by telephone directly or through a nominated friend or relative, were recruited. ${ }^{18}$ Participants were ineligible based on any of the following conditions at baseline assessment: (1) participant or family member was taking potassium-sparing diuretic; (2) participant or family member was taking potassium supplement; (3) participant or family member had serious renal impairment; (4) participant or family member had other reason for concern about using salt substitute; (5) participant ate most meals outside the home; (6) participant was not expected to live longer than 6 months from the date of baseline assessment by the village doctor. ${ }^{18}$

\section{Intervention and control}

After participant enrolment and completion of the baseline survey, randomisation was conducted through stratification at the county level and a 1:1 allocation of villages to the salt substitution programme or control. Participants in the intervention villages were provided with reducedsodium salt substitute for free to replace all use of regular salt at home among the whole family. Participants were also recommended to minimise the total amount of salt substitute used and provided oral, written booklets and other reminders (eg, cooking apron) to promote the potential benefits of using salt substitute. In the control villages, participants continued their use of regular salt and were provided with advice about stroke prevention and salt reduction only at trial commencement.

\section{Economic evaluation}

A within-trial economic evaluation of the SSaSS will be performed from a healthcare system perspective, considering costs and outcomes relevant to future policymaking, for example, subsidy for salt substitute. A costeffectiveness analysis (CEA) and cost-utility analysis (CUA) will be conducted outlining the changes in outcomes and costs between the intervention and control conditions over the trial period. ${ }^{19}$ The resulting incremental costeffectiveness ratio will be expressed as cost per cardiovascular event averted for CEA and cost per quality-adjusted life year (QALY) gained for CUA. Cost and outcomes will be discounted using 5\% annual rate following Chinese guidelines for pharmacoeconomic evaluations. ${ }^{20}$

\section{Measure of outcome}

The primary measure of effectiveness for CEA will be stroke and other major adverse cardiac events averted. This information was collected every 6 months through face-to-face interview with participants in the first 2 years; then through routinely collected data from the health insurance registry as well as the mortality surveillance system. Additionally, all the events collected were adjudicated by an independent endpoint event adjudication committee with support from medical records.

Health-related quality of life was measured using the EuroQol-5-Dimensions (EQ-5D-5L). The questionnaire comprises five dimensions: mobility, self-care, usual activities, pain/discomfort and anxiety/depression, and each dimension is described at five levels: no, slight, 
moderate, severe and extreme problems. ${ }^{21}$ Alongside the process indicators survey, EQ-5D-5L surveys were completed by a subset of the trial population at baseline, 12-month, 24-month, 36-month, 48-month follow-up; and by all alive participants at end-of-trial (60-month) follow-up. Responses to the EQ-5D-5L will be scored using preference value sets developed for the Chinese population, ${ }^{21}$ which will convert the five responses into a single preference-based index value, where a score of 1 represents perfect health and 0 indicates the dead health state. ${ }^{22}$ For participants who did not take part in the survey at each measurement point, EQ-5D-5L values will be imputed using a matching process based on gender, age, province and history of disease. QALYs gained over the trial period will be estimated for each individual by calculating the area under the quality of life curve.

\section{Measure of cost and resource use}

Costs in both trial arms will be estimated from a healthcare system perspective, using a combination of trial and administratively linked data records. The salt substitute was provided free of charge for each participant's household in the trial, however, in consideration of the real-world setting, the cost of salt substitute will be incorporated into the analysis. Costs of the salt substitute will be calculated according to market prices and mean population daily salt usage amount. Healthcare costs associated with inpatient and outpatient services will be collected using data from the New Rural Cooperative Medical Scheme (NCMS). The NCMS is the universal health insurance programme for rural residents in China and began in 2003. In 2012, its coverage reached 98\% of the rural population and the average reimbursement rates for inpatient and outpatient service were $55 \%$ and $50 \%$, respectively. ${ }^{23}$ Routinely collected data from the NCMS provide detailed information such as diagnosis, reimbursement and out-of-pocket payment for both inpatient and outpatient services at all levels. Each participant's aggregate service use cost of relevant outcomes will be valued from the total of instances of service use over the duration of the trial period.

\section{Sensitivity analysis}

Sensitivity analysis will be undertaken to explore the impact of changes on different parameters to the robustness of the cost-effectiveness results. Changes in the cost of the salt substitute, discount rate $(0 \%-8 \%),{ }^{20}$ healthcare costs and health-related quality of life will be explored using one-way sensitivity analysis. Bootstrapping at an individual level will be conducted to estimate the joint uncertainty in the distribution of costs and effectiveness and depicted in a cost-effectiveness plane. A cost-effectiveness acceptability curve ${ }^{24}$ will be presented to show the probability of cost-effectiveness of the bootstrapped samples for a range of willingness-to-pay values.

\section{Patient and public involvement}

No patient involved.

\section{Approvals}

The SSaSS trial received ethics approval from the University of Sydney Ethics Committee (2013/888) and Peking University Institutional Review Board (IRB0000105213069). The trial was registered in the ClinicalTrials.gov database on 12 March 2014.

\section{DISCUSSION}

This study will evaluate the cost-effectiveness of salt reduction via the use of a salt substitute alongside a large cluster randomised trial in China. SSaSS is so far the biggest trial to look at the effects of salt reduction on the risk of CVD around the world and the results will address the gap of scientific evidence significantly. Existing economic evaluation studies of salt substitution primarily focused on the blood pressure effects, while, to the best of our knowledge, this is the first economic evaluation assessing the effects of salt substitution on morbidity and mortality of CVD. Furthermore, routinely collected data from the NCMS that effectively provide longitudinal records for individuals will be used in estimation of the medical costs.

A potential limitation is that the increased compliance in using salt substitute observed in the trial may not reflect the counterparts in a real-world setting. Also, NCMS does not reach $100 \%$ coverage and participants may drop out from the NCMS. However, the missing events can be complemented by the face-to-face follow-up. In addition, most of quality of life data were collected from a random subset $(10 \%$ of clusters) of trial population during the four mid-term follow-ups. Nevertheless, the collected data will be compared with utility values from different sources of literature, which will inform the imputation strategy for participants without the data. These limitations might potentially increase the uncertainty around the effect size found in the trial.

Overall, this project has the potential to determine whether the use of a sodium-reduced, potassiumenriched salt substitute in daily life can be cost-effective in the prevention of stroke and major CVDs for a high-risk population. Evidence of cost-effectiveness can be used to advocate for a relevant and effective salt subsidy policy and the promotion of this new salt reduction strategy to other countries or areas with similar public health issues.

\section{Author affiliations}

${ }^{1}$ The George Institute for Global Health, University of New South Wales, Sydney, New South Wales, Australia

${ }^{2}$ The George Institute for Global Health at Peking University Health Science Center, Beijing, China

${ }^{3}$ School of Public Health, Imperial College London, London, UK

${ }^{4}$ Department of Cardiology, The Third Affiliated Hospital of Peking University Health Science Center, Beijing, China

${ }^{5}$ Peking University Clinical Research Center, Peking University, Beijing, China

${ }^{6}$ George Clinical, Sydney, New South Wales, Australia

${ }^{7}$ Global Health Research Center, Duke Kunshan University, Kunshan, China

${ }^{8}$ Feinberg School of Medicine, Northwestern University, Chicago, Illinois, USA

${ }^{9}$ Department of Evidence-based Medicine, First Hospital of China Medical University,

Shenyang, China

${ }^{10}$ School of Public Health, Changzhi Medical College, Changzhi, China 
${ }^{11}$ Department of Non-communicable Disease Prevention and Control, Center for Disease Control of Hebei Province, Shijiazhuang, China

${ }^{12}$ School of Public Health, Ningxia Medical University, Yinchuan, China

${ }^{13}$ School of Public Health, Xi'an Jiaotong University, Xi'an, China

${ }^{14}$ Sydney School of Public Health, University of Sydney, Sydney, New South Wales, Australia

\section{Twitter Maoyi Tian @MaoyiT}

Acknowledgements The SSaSS implementation team (Ms Yanqing Wang, Ms Ying Cai, Ms Lili Wang, Ms Baoyu Shan, Mr Tianqi Hu, Ms Yang Shen and Ms Zhuo Meng) and local collaborators. Endpoint Adjudication Committee: Dr Weiping Sun, Dr Junyan Liu, Dr Fang Liu, Dr Yun Jiang, Dr Rong Hu, Dr Yong Peng, Dr Jiehui Shan and Dr Jie Yu. Data Monitoring Committee: Professor Sir Rory Collins (Chair), Professor Zhengming Chen, Professor Jonathan Emberson, Professor Peter Sandercock, Professor Paul Whelton and Ms Sandrine Stepien.

Contributors K-CL, TL and LS conceived the protocol for economic evaluation. MT and BN provided guidance from the trial perspective. $\mathrm{K}$-CL wrote the first draft of the manuscript. MT, BN, YW, NL, PE, LY and DL contributed to conceptualisation and funding acquisition of the trial. $\mathrm{LH}, \mathrm{JY}, \mathrm{YL}, \mathrm{XY}, \mathrm{XZ}$ and $\mathrm{K}-\mathrm{CL}$ contributed to data curation of the trial. MT, ZH, JShi, XF, JZ, YZhang, RZ, BZ, ZL, JSun, YZhao and YY contributed to local implementation, investigation and supervision of the trial. All authors commented on the further drafts and approved the final version.

Funding The SSaSS trial is funded by Australian National Health and Medical Research Council (APP 1049417 \& APP1164206). K-CL and XY are both supported by the UNSW Scientia PhD scholarship. YL is supported by the university international postgraduate award of UNSW. LS is supported by an NHMRC Early Career Fellowship (GNT1139826). TL is supported by an NHMRC Early Career Fellowship (APP1141392) and National Heart Foundation Postdoctoral Fellowship (101956)

Competing interests Between 24 months and 48 months, salt substitute in SSaSS has been provided by Jiangsu Sinokone Technology Co for free.

Patient and public involvement Patients and/or the public were not involved in the design, or conduct, or reporting, or dissemination plans of this research.

Patient consent for publication Not required.

Provenance and peer review Not commissioned; externally peer reviewed.

Open access This is an open access article distributed in accordance with the Creative Commons Attribution Non Commercial (CC BY-NC 4.0) license, which permits others to distribute, remix, adapt, build upon this work non-commercially, and license their derivative works on different terms, provided the original work is properly cited, appropriate credit is given, any changes made indicated, and the use is non-commercial. See: http://creativecommons.org/licenses/by-nc/4.0/.

\section{ORCID iDs}

Ka-Chun Li http://orcid.org/0000-0002-5788-528X

Jie Yu http://orcid.org/0000-0002-7401-7580

Xuejun Yin http://orcid.org/0000-0001-8446-9591

Paul Elliott http://orcid.org/0000-0002-7511-5684

Lei Si http://orcid.org/0000-0002-3044-170X

Thomas Lung http://orcid.org/0000-0001-9978-6311

\section{REFERENCES}

1 Roth GA, Abate D, Abate KH, et al. Global, regional, and national age-sex-specific mortality for 282 causes of death in 195 countries and territories, 1980-2017: a systematic analysis for the global burden of disease study 2017. Lancet 2018;392:1736-88.

2 James SL, Abate D, Abate KH, et al. Global, regional, and national incidence, prevalence, and years lived with disability for 354 diseases and injuries for 195 countries and territories, 1990-2017: a systematic analysis for the global burden of disease study 2017. Lancet 2018;392:1789-858.

3 Foreman KJ, Marquez N, Dolgert A, et al. Forecasting life expectancy, years of life lost, and all-cause and cause-specific mortality for 250 causes of death: reference and alternative scenarios for 2016-40 for 195 countries and territories. Lancet 2018;392:2052-90.

4 World Health Organization. A global brief on hypertension, 2013.

5 Ettehad D, Emdin CA, Kiran A, et al. Blood pressure lowering for prevention of cardiovascular disease and death: a systematic review and meta-analysis. Lancet 2016;387:957-67.

6 GBD 2017 Diet Collaborators. Health effects of dietary risks in 195 countries, 1990-2017: a systematic analysis for the global burden of disease study 2017. Lancet 2019;393:1958-72.

7 He FJ, Li J, Macgregor GA. Effect of longer term modest salt reduction on blood pressure: cochrane systematic review and metaanalysis of randomised trials. BMJ 2013;346:f1325-f25.

8 Graudal NA, Hubeck-Graudal T, Jurgens G. Effects of low sodium diet versus high sodium diet on blood pressure, renin, aldosterone, catecholamines, cholesterol, and triglyceride. Cochrane Database Syst Rev 2017:4:CD004022.

9 Oparil S. Low sodium intake--cardiovascular health benefit or risk? $N$ Engl J Med 2014;371:677-9.

10 Mente A, O'Donnell MJ, Dagenais G, et al. Validation and comparison of three formulae to estimate sodium and potassium excretion from a single morning fasting urine compared to 24-h measures in 11 countries. J Hypertens 2014;32:1005-15.

$11 \mathrm{Li} \mathrm{N}$, Prescott J, Wu Y, et al. The effects of a reduced-sodium, highpotassium salt substitute on food taste and acceptability in rural Northern China. Br J Nutr 2009;101:1088-93.

12 Zhao X, Yin X, Li X, et al. Using a low-sodium, high-potassium salt substitute to reduce blood pressure among Tibetans with high blood pressure: a patient-blinded randomized controlled trial. PLoS One 2014;9:e110131.

13 Schorling E, Niebuhr D, Kroke A. Cost-effectiveness of salt reduction to prevent hypertension and CVD: a systematic review. Public Health Nutr 2017;20:1993-2003.

14 Webb M, Fahimi S, Singh GM, et al. Cost effectiveness of a government supported policy strategy to decrease sodium intake: global analysis across 183 nations. BMJ 2017;356:6699.

15 Nghiem N, Blakely T, Cobiac LJ, et al. Health and economic impacts of eight different dietary salt reduction interventions. PLoS One 2015;10:e0123915.

16 Bernabe-Ortiz A, Sal Y Rosas VG, Ponce-Lucero V, et al. Effect of salt substitution on community-wide blood pressure and hypertension incidence. Nat Med 2020;26:374-8.

17 Marklund M, Singh G, Greer R, et al. Estimated population wide benefits and risks in China of lowering sodium through potassium enriched salt substitution: modelling study. BMJ 2020;369:m824.

18 Neal B, Tian M, Li N, et al. Rationale, design, and baseline characteristics of the Salt Substitute and Stroke Study (SSaSS)-A large-scale cluster randomized controlled trial. Am Heart $J$ 2017;188:109-17.

19 Drummond MF, Sculpher MJ, Claxton K. Methods for the economic evaluation of health care programmes. Oxford university press, 2015

20 China guidelines for pharmacoeconomic evaluations, 2011. Available: https://tools.ispor.org/PEguidelines/countrydet.asp?c=28\& $\mathrm{t}=4$ [Accessed 20 Aug 2020].

21 Luo N, Liu G, Li M, et al. Estimating an EQ-5D-5L value set for China Value Health 2017;20:662-9.

22 Ramos-Goñi J, Oramas-Zarate J, Rivero-Arias O. eq5d5I: a command to estimate preference-based values, 2019.

23 Hsiao W, Li M, Zhang S. Universal health coverage: the case of China. UNRISD working paper. United Nations Research Institute for Social Development (UNRISD), 2015.

24 Fenwick E, Claxton K, Sculpher M. Representing uncertainty: the role of cost-effectiveness acceptability curves. Health Econ 2001;10:779-87. 